\title{
Long-term Clinical Outcomes for Overnight Corneal Reshaping in Children and Adults
}

\author{
Michael J. Lipson, O.D., F.A.A.O.
}

Purpose. To retrospectively evaluate outcomes of overnight corneal reshaping (OCR) in children aged 12 years or younger compared to children older than 12 years and adults at one practice to establish the efficacy and safety of OCR during a period of 51 months. Methods. Examination records of OCR patients were reviewed for pretreatment data, including manifest refraction, keratometric readings, topography, corneal staining, and age at beginning OCR. Posttreatment records were reviewed for manifest refraction, unaided visual acuity, keratometric readings, topography, corneal staining, adverse events, and duration of OCR lens wear. Results. Records of 296 OCR patients were evaluated. One hundred fifty-four $(52.0 \%)$ patients were 12 years old or younger. Sixty-eight percent of all patients in the study were Asian, and almost $95 \%$ of the patients aged 12 years or younger were Asian. The patients aged 12 years or younger had a mean original spherical equivalent refractive error of $-3.50 \pm 1.50$ diopters (D). The patients older than 12 years had a mean original spherical equivalent refractive error of $-3.20 \pm 1.50 \mathrm{D}$. Refractive changes were similar between the group aged 12 years or younger and the group older than 12 years $(3.30 \pm 1.40 \mathrm{D}$ vs. $3.10 \pm 1.40 \mathrm{D})$ $(P=0.14)$. The mean unaided, binocular $\log$ MAR visual acuity was $0.03 \pm 0.06$ (i.e., 20/20 $0^{-1}$ ) for the group aged 12 years or younger and $0.02 \pm 0.07$ (i.e., $20 / 20^{-1}$ ) for the group older than 12 years. There were three adverse events during the study that did not result in a loss of best-corrected visual acuity. A total of 507 patient-years of wear was represented in the study. Conclusions. OCR resulted in comparable safety and efficacy in temporarily reducing myopia for children younger than 12 years as it is for children older than 12 years and adults.

Key Words: Corneal refractive therapy-Myopia reduction in children-Overnight corneal reshaping-Refractive error-Retrospective study.

Overnight corneal reshaping (OCR) is an alternative method to correct myopia in adults and children. ${ }^{1,2}$ OCR involves specially designed, reverse-geometry, rigid gas-permeable contact lenses worn while sleeping to temporarily reduce myopia by reshaping the corneal epithelium. The lenses are made of highly permeable materials in specific designs that have been approved by the Food and Drug Administration ${ }^{3,4}$ for overnight wear. OCR has been studied to analyze cellular changes of the cornea, ${ }^{5-8}$ evaluated for changes in visual performance and optical quality, ${ }^{9-12}$ corneal

From the Department of Ophthalmology and Visual Sciences, University of Michigan, MI.

Address correspondence and reprint requests to Dr. M.J. Lipson, 19900 Haggerty Rd. \#111, Livonia, MI 48152.

Accepted May 16, 2007.

DOI: 10.1097/ICL.0b013e31811eba10 curvature, refraction, and unaided visual acuity, ${ }^{13,14}$ and evaluated for its effect on vision-related quality of life. ${ }^{15,16}$ One crossover study with OCR and soft disposable lenses showed that $85 \%$ of patients with myopia between -1.00 and -3.00 diopters (D) preferred OCR. ${ }^{15}$ Studies have also shown that the corneal and refractive changes made during OCR are reversible. ${ }^{17,18}$ Furthermore, studies have looked specifically at the efficacy of OCR for children and have shown the process to be effective in temporarily changing the cornea to reduce myopia. ${ }^{1,19,20}$ OCR has also been compared to LASIK for effectiveness and patient satisfaction. ${ }^{21}$ These cited studies have shown OCR to be effective in temporarily reducing myopia with little or no mention of any adverse events. Studies have shown that wearing soft contact lenses while sleeping is associated with an increased risk of microbial keratitis. ${ }^{22}$ To date, there are no studies to evaluate such a risk with OCR lens wear. ${ }^{23}$ There are a number of case reports of serious complications, such as microbial keratitis in children and adult patients using OCR lenses. ${ }^{24-30}$

The purpose of this study was to retrospectively examine the results of OCR by a single doctor for efficacy and safety. The findings are reported for adults and children during a 4-year period and compare pretreatment data with outcomes for myopia reduction, unaided visual acuity, corneal staining, and adverse events. Previous studies on OCR have been conducted with a small study population (i.e., 50 or fewer subjects) and for short periods (i.e., less than 1 year). ${ }^{13,23}$ This study has a significantly greater population monitored for a longer period. Retrospective analyses such as this have been recommended by concerned groups of eye care professionals and regulators. ${ }^{24,31,32}$ It is not known at this time whether the risk of adverse events with OCR is different with children versus adults. ${ }^{24,31}$ As the process of contact lens corneal reshaping becomes more widely used, information on efficacy and safety is critical for doctors, patients, and parents to assess individual patient characteristics that influence potential success and risks before commencing treatment. ${ }^{32}$

\section{MATERIALS AND METHODS}

Records of 296 OCR patients were reviewed for data before and during treatment between May 2002 and August 2006. Included in this study were all patients of any age who were seen for fitting or care involving corneal reshaping. Approval from the University of Michigan's Institutional Review Board was obtained to conduct this retrospective review of records. Most patients were first-time wearers fitted and followed up by the author. The population included patients who were previously fitted by other practitioners who were seen for refitting, modification, or continuation of care. 
Baseline examination included a comprehensive pretreatment vision and eye health evaluation, including unaided visual acuity, acuity with current spectacles, keratometry, topography, manifest refraction, phoria and cover testing, tonometry, slitlamp examination, and fundus examination. If patients were good candidates for OCR and they chose to wear OCR lenses, a diagnostic evaluation with OCR lenses was performed. After a successful diagnostic evaluation, patients or their parents signed an OCR informed consent and fitting agreement, and lenses were ordered. OCR lenses were dispensed during a session of careful instruction on insertion, removal, and care of lenses.

Initial follow-up visits were routinely performed after the first night of wear, 1 week, 1 month, 2 months, 4 months, and 6 months. After the 6-month visit, follow-up care included an annual comprehensive examination and a checkup every 6 months or additional visits as necessary for symptomatic reports of discomfort, change in vision, or damaged or lost lenses. The during-treatment data used in the study included manifest refraction, best-corrected visual acuity, slitlamp observations with grading of findings, and unaided visual acuity. All visual acuity measurements were performed monocularly and binocularly with a high-contrast acuity chart with Snellen notation converted to logMAR for statistical purposes. Refractive data for refractive change was calculated from baseline to the most recent follow-up visit. Evaluations made for slitlamp findings of corneal staining were recorded for degree and location. Baseline (pretreatment) evaluation data were available for 282 patients. Grade changes were defined as a change from baseline grade to any other visit of "none to trace, $1+$, or $2+$ " or a change from "trace to $1+$ or $2+$." Data were also tracked on age at commencement of OCR, months of OCR lens wear, whether patients were still currently using OCR lenses, and reporting of adverse events or complications during OCR lens wear. Corneal topography was obtained at each follow-up visit to ensure proper fitting and corneal integrity but was not used for data analysis.

Overnight corneal reshaping lenses used in the study were Paragon CRT (Paragon Vision Sciences, Mesa, AZ) or customdesigned lenses made from Boston XO material (Polymer Technology/Bausch \& Lomb, Rochester, NY) manufactured by Art Optical Contact Lens, Inc. (Grand Rapids, MI). Recommended care solutions were Unique $\mathrm{pH}$ (Alcon, Fort Worth, TX) or Boston Simplus (Bausch \& Lomb, Rochester, NY).

\section{Definition of an Adverse Event}

In this study, an adverse event included any of the following: microbial keratitis or a corneal ulcer, a corneal abrasion requiring medical treatment, loss of best-corrected visual acuity, or a corneal scar.

Data were analyzed by using SAS 9.1 statistical software (SAS Institute, Cary, NC). Comparisons between age groups made use of independent, two-tailed Student $t$ tests. Comparisons of change from baseline findings to post-OCR findings were performed with paired Student $t$ tests. Comparisons of duration of OCR lens wear were performed with Mann-Whitney $U$ tests. Comparisons of proportions (e.g., ethnicity percentage) made use of the chi-square test.

\section{RESULTS}

Data from the records of 296 OCR patients were analyzed in the study. The mean age of all patients at the commencement of OCR

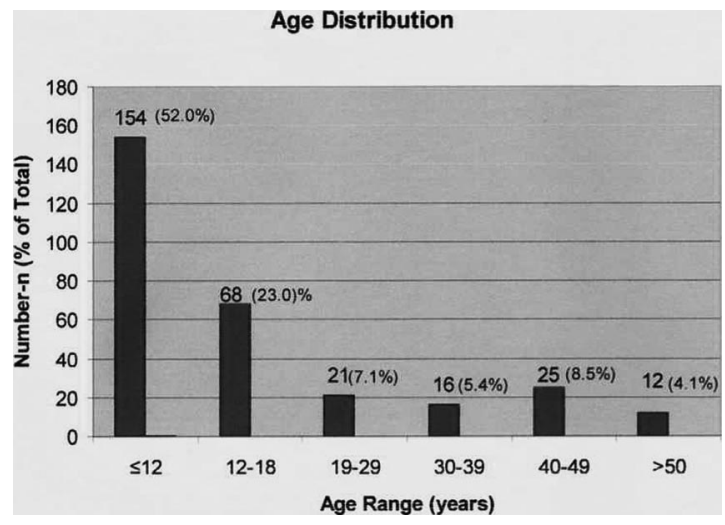

FIG. 1. Age distribution.

was $17.7 \pm 13.2$ years. One hundred fifty-four $(52.0 \%)$ patients were 12 years and 0 months old or younger at the commencement of OCR, and $142(47.9 \%)$ patients were 12 years and 1 month old or older (Fig. 1). Of the 296 total patients, 146 (49.3\%) were male and $150(50.3 \%)$ were female. The mean duration of wear was $21.6 \pm 18.1$ months. For patients aged 12 years or younger, the mean duration of wear was $20.8 \pm 14.7$ months, whereas the mean duration of wear for those patients older than 12 years was $22.5 \pm$ 21.2 months. The difference in duration of wear between the two groups was not significant $(P=0.6462)$. This represents a total of 506.95 patient-years of wear during the 51 months (i.e., 4.25 years) of the study period.

\section{Ethnicity}

Two hundred one (68\%) patients were Asian; 90 (30\%) were white; three $(1 \%)$ were black; and two $(<1 \%)$ were Eastern Indian.

Of the 154 patients aged 12 years or younger, $146(94.8 \%)$ were Asian and eight (5.2\%) were of other ethnicity. Of the 142 patients older than 12 years, 55 (38.7\%) were Asian and 87 (61.3\%) were of other ethnicity. The difference in ethnic distribution between the two groups was statistically significant $(P<0.0001)$.

\section{Termination of OCR Lens Wear}

During the study, $32(10.8 \%)$ patients discontinued OCR lens wear (Table 1). Of those 32, only six were aged 12 years or younger (6 [3.9\%] of the 154), whereas 26 were older than 12 years (26 [18.3\%] of the 142). It is statistically significant that $96.1 \%$ of those aged 12 years or younger are still using OCR lenses, whereas $81.7 \%$ of those older than 12 years are still wearing OCR lenses $(P<0.0001)$.

TABLE 1. Reasons for Overnight Corneal Reshaping Discontinuation

\begin{tabular}{lccc}
\hline Reason & No. & $\begin{array}{c}\text { Aged 12 years } \\
\text { or younger }\end{array}$ & $\begin{array}{c}\text { Older than } \\
\text { 12 years }\end{array}$ \\
\hline Suboptimal visual acuity & 4 & 1 & 3 \\
Comfort & 7 & 1 & 6 \\
High refractive error (>-4.75 D) & 6 & 3 & 3 \\
Solution reaction & 1 & 0 & 1 \\
Handling or inconvenience & 3 & 0 & 3 \\
Dry eye & 1 & 0 & 1 \\
Underwent LASIK & 2 & 0 & 2 \\
Preferred soft contact lenses & 5 & 0 & 5 \\
Moved & 3 & 1 & 2 \\
Total & 32 & 6 & 26 \\
\hline
\end{tabular}


Refractive Change By Age

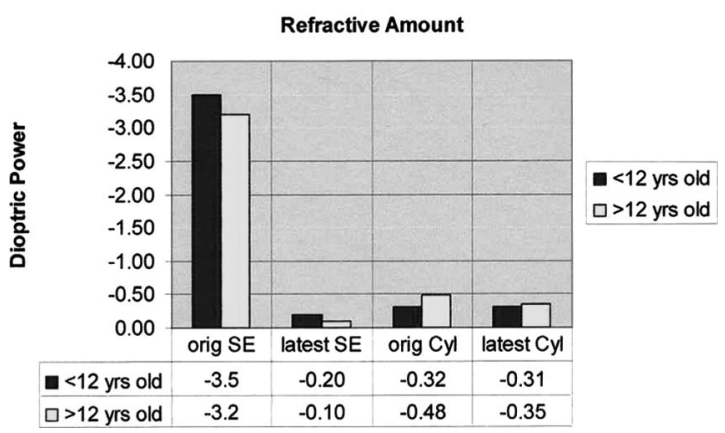

FIG. 2. Refractive change by age.

\section{Refractive Error}

The mean original spherical equivalent refractive error for all patients and all eyes was $-3.40 \pm 1.5 \mathrm{D}$, and the mean original cylinder was $-0.42 \pm 0.46 \mathrm{D}$. In the group aged 12 years or younger, the mean original spherical equivalent refractive error was $-3.50 \pm$ $1.5 \mathrm{D}$, and the mean original cylinder was $-0.33 \pm 0.36 \mathrm{D}$. In the group older than 12 years, the mean original spherical equivalent refractive error was $-3.20 \pm 1.5 \mathrm{D}$, and the mean original cylinder was $-0.49 \pm 0.55 \mathrm{D}$ (Fig. 2). At the latest examination, the mean spherical equivalent for all eyes had reduced to $-0.20 \pm 0.40 \mathrm{D}$, resulting from a mean change in the spherical equivalent of $3.20 \pm$ $1.4 \mathrm{D}$. Because the target result was a spherical equivalent of $0.00 \mathrm{D}$, the mean change in spherical equivalent was greater for the group with the higher mean original spherical equivalent. The mean spherical equivalent change for the group aged 12 years or younger was $3.30 \pm 1.4 \mathrm{D}$ versus $3.10 \pm 1.4 \mathrm{D}$ for the group older than 12 years $(P=0.1385)$.

The mean original cylinder amount was $-0.40 \pm 0.47 \mathrm{D}$, which was reduced at the latest examination by $0.07 \pm 0.41 \mathrm{D}$ to -0.33 $\pm 0.41 \mathrm{D}(P=0.0039)$. For the group aged 12 years or younger, the mean original cylinder was $-0.32 \pm 0.34 \mathrm{D}$, which decreased at the latest examination by $0.01 \pm 0.35 \mathrm{D}$ to $-0.31 \pm 0.35 \mathrm{D}$. For the group older than 12 years, the mean original cylinder was $-0.48 \pm 0.57 \mathrm{D}$, which decreased at the latest examination by $0.13 \pm 0.46 \mathrm{D}$ to $-0.35 \pm 0.33 \mathrm{D}$. The difference between the amount of cylinder change in the two age groups was significant $(P=0.02)$.

\section{Keratometric Readings}

The mean flat keratometric reading for all eyes was $43.37 \pm 1.4$ D. The mean flat keratometric reading was $43.12 \pm 1.5 \mathrm{D}$ for the group aged 12 years or younger and $43.59 \pm 1.4 \mathrm{D}$ for the group older than 12 years. Comparison of the flat keratometric reading at baseline and unaided visual acuity or refractive change at the latest examination showed no significant relationship.

\section{Visual Acuity}

At the latest examination, the mean unaided distance visual acuity for all patients was $0.08 \pm 0.10$ (i.e., 20/25 $5^{+1}$ ) for the right eye, $0.08 \pm 0.10$ for the left eye, $0.03 \pm 0.07$ (i.e., $20 / 20^{-1}$ ) for both eyes, and $0.11 \pm 0.12$ (i.e., 20/25) for the worse eye. The mean unaided distance visual acuity for the group aged 12 years or younger was $0.08 \pm 0.08$ for the right eye, $0.08 \pm 0.08$ for the left eye, $0.03 \pm 0.06$ for both eyes, and $0.10 \pm 0.08$ for the worse eye. For the group older than 12 years, the mean unaided distance
TABLE 2. Unaided Acuity Distribution

\begin{tabular}{|c|c|c|c|c|c|c|}
\hline \multirow[b]{2}{*}{$\begin{array}{l}\text { Unaided } \\
\text { logMAR } \\
\text { visual acuity }\end{array}$} & \multicolumn{3}{|c|}{ Aged 12 years or younger } & \multicolumn{3}{|c|}{ Older than 12 years } \\
\hline & $\begin{array}{c}\text { Right } \\
\text { eye, } \\
\text { no. (\%) }\end{array}$ & $\begin{array}{c}\text { Left } \\
\text { eye, } \\
\text { no. (\%) }\end{array}$ & $\begin{array}{c}\text { Both } \\
\text { eyes, } \\
\text { no. (\%) }\end{array}$ & $\begin{array}{c}\text { Right } \\
\text { eye, } \\
\text { no. (\%) }\end{array}$ & $\begin{array}{c}\text { Left } \\
\text { eye, } \\
\text { no. (\%) }\end{array}$ & $\begin{array}{c}\text { Both } \\
\text { eyes, } \\
\text { no. (\%) }\end{array}$ \\
\hline-0.01 to -0.10 & $3(2.0)$ & $1(0.7)$ & $28(18.2)$ & $5(3.5)$ & $3(2.1)$ & $31(21.8)$ \\
\hline 0.0 to 0.09 & $87(56.5)$ & $83(54.3)$ & $97(63.0)$ & $89(62.7)$ & $83(53.5)$ & $89(62.7)$ \\
\hline 0.1 to 0.19 & $54(35.1)$ & 59 (38.6) & 27 (17.5) & 34 (23.9) & 41 (28.9) & $18(12.7)$ \\
\hline 0.2 to 0.29 & $9(5.8)$ & $6(3.9)$ & $2(1.3)$ & $8(5.6)$ & $5(3.5)$ & 2 (1.4) \\
\hline 0.3 to 0.39 & $1(0.7)$ & $4(2.6)$ & $0(0.0)$ & $3(2.1)$ & $6(4.2)$ & $2(1.4)$ \\
\hline
\end{tabular}

visual acuity was $0.07 \pm 0.11$ (i.e., $20 / 20^{-3}$ ) for the right eye, $0.09 \pm 0.13$ (i.e., $20 / 25^{+1}$ ) for the left eye, $0.02 \pm 0.07$ (i.e., $20 / 20^{-1}$ ) for both eyes, and 0.11 (i.e., $20 / 25^{-1}$ ) for the worse eye. For the group aged 12 years or younger, $93.6 \%$ had a monocular unaided visual acuity of 0.19 or less (i.e., better than 20/30) in each eye (Table 2). For the group older than 12 years, $89.8 \%$ had a monocular visual acuity of 0.19 or less. For the group aged 12 years or younger, $98.7 \%$ had a visual acuity of 0.19 or better, and $97.2 \%$ of the group older than 12 years had a visual acuity of 0.19 or better.

\section{Slitlamp Findings}

Corneal staining was evident in $25(8.8 \%)$ of 282 of the patients at the baseline examination. All were graded trace; 14 cases were peripheral (i.e., exposure or incomplete blinking); and 11 were the result of misdirected eyelashes (i.e., trichiasis). Corneal staining was observed in 90 (32\%) of the 282 patients at the 1-day examination, in $45(16 \%)$ of the patients at the 1-week visit, in 15 $(5.3 \%)$ of the patients at the 1-month visit, and in $75(25.3 \%)$ of 296 patients at any other visit during the study (Table 3 ).

Multivariable analysis showed some significant findings. When comparing baseline findings to all follow-up visits, $58.2 \%$ of patients had no change in or less staining and $41.8 \%$ had increased staining at one or more visits. Patients who showed an increase in staining versus baseline had a higher original spherical equivalent $(-3.70$ D vs. -3.11 D) $(P=0.0013)$ than did those who showed no change in staining. In comparing increased grade of staining between lenses of different designs, a higher percentage of patients showed an increased grade of staining with the custom-designed lenses than the CRT lenses $(52 \%$ vs. $36 \%)(P=0.0104)$.

Trends observed in this same analysis were that younger children showed a trend toward more staining increase than older children and that there was no central staining observed at baseline. Central staining was most often observed after the first night of wear (26.6\%) and least often at the 1-month visit (2.1\%).

TABLE 3. Corneal Staining

\begin{tabular}{cccccc}
\hline & $\begin{array}{c}\text { Baseline } \\
(n=282)\end{array}$ & $\begin{array}{c}\text { 1-day } \\
\text { visit } \\
(n=282)\end{array}$ & $\begin{array}{c}\text { 1-week } \\
\text { visit } \\
(n=282)\end{array}$ & $\begin{array}{c}\text { 1-month } \\
\text { visit } \\
(n=282)\end{array}$ & $\begin{array}{c}\text { Other } \\
\text { visit } \\
(n=296)\end{array}$ \\
\hline Central trace & 0 & 57 & 26 & 6 & 37 \\
Central 1+ & 0 & 15 & 2 & 0 & 8 \\
Central 2+ & 0 & 3 & 0 & 0 & 3 \\
Peripheral trace & 14 & 6 & 11 & 5 & 13 \\
Peripheral 1+ & 0 & 1 & 0 & 0 & 1 \\
Peripheral 2+ & 0 & 1 & 0 & 0 & 2 \\
Trichiasis trace & 11 & 6 & 5 & 3 & 8 \\
Trichiasis 1+ & 0 & 1 & 1 & 1 & 2 \\
Trichiasis 2+ & 0 & 0 & 0 & 0 & 1 \\
\hline
\end{tabular}




\section{Safety and Adverse Events}

During the study, three patients had an incident of an adverse event, as defined earlier. Each of the adverse events was central, in children aged 12 years or younger, and after a minimum of 6 months of OCR lens wear. None of the events resulted in a loss of best-corrected visual acuity and each of the patients has continued to wear OCR lenses. One patient had total resolution, and the other two have central scarring remaining.

\section{Lens Type}

Of the two different lens designs, Paragon CRT and customdesigned reverse-geometry lenses in Boston XO material, 192 (65\%) patients wore the CRT lenses and 104 (35\%) wore the custom lenses. By age group, $111(72 \%)$ of the group aged 12 years or younger wore the CRT lenses, whereas $43(28 \%)$ wore the custom lenses. In the group older than 12 years, 81 (57\%) wore the CRT lenses and $61(43 \%)$ wore the custom lenses. These are significant differences between the two age groups $(P=0.0068)$. Patients wearing the custom lenses showed a higher original spherical equivalent $(-4.00 \pm 1.7 \mathrm{D}$ vs. $-3.05 \pm 1.3 \mathrm{D}$ for the CRT wearers $[P<0.0001])$. The custom lens-wearing patients also had a statistically significantly higher original cylinder $(0.56 \pm$ $0.59 \mathrm{D}$ vs. $0.33 \pm 0.35 \mathrm{D}$ for the CRT wearers $[P<0.0001])$. Because of the targeted refractive change in OCR, analysis also showed a significantly higher amount of spherical equivalent change in the custom lens wearers than in the CRT wearers (3.70 $\pm 1.6 \mathrm{D}$ vs. $2.90 \pm 1.3 \mathrm{D})$. However, there was also a greater cylinder change (i.e., decreased cylinder power) in the custom lens wearers $(0.17 \pm 0.48 \mathrm{D}$ vs. $0.03 \pm 0.36 \mathrm{D}$ for the CRT wearers).

Mean binocular unaided visual acuity was $0.02 \pm 0.07$ for the CRT wearers and $0.04 \pm 0.07$ for the custom lens wearers. Of the CRT wearers, $98.5 \%$ had an unaided binocular visual acuity of 0.19 or less (i.e., 20/30 or better). Of the custom lens wearers, $97.1 \%$ had an unaided binocular visual acuity of 0.19 or less.

\section{DISCUSSION}

Use of OCR has increased in the United States and worldwide in recent years. This study objectively evaluated clinical outcomes of refractive changes, visual acuity, and corneal health for safety and efficacy for more than 4 years. The findings establish that vision correction is good and that there were isolated minor complications. The results show some important findings regarding differences in refractive changes and age.

\section{Age Differences}

As noted in the results, $52 \%$ of the study patients were aged 12 years or younger. Because there was slightly higher original refractive error in the younger patients, there was a correspondingly higher refractive change in the group aged 12 years or younger. It is also noteworthy that this group had a slightly flatter flat keratometric reading at baseline, but showed a slightly higher degree of myopia. This suggests that the mostly Asian population of patients aged 12 years or younger had a greater axial length of the eye (not evaluated in this study). Patients in both age groups achieved targeted vision improvement, and unaided visual acuity was not significantly different between the two age groups. Overall, unaided visual acuity was excellent, and patients often commented about their good quality of vision.

\section{Corneal Staining}

Corneal staining was a common observation in patients during the study. Low grade corneal staining has also been documented in as many as $79 \%$ of non-contact lens wearers and in $33 \%$ to $55 \%$ of asymptomatic soft lens wearers. ${ }^{33-36}$ The incidence of corneal staining observed in this study is similar to that in soft lens studies and shows that OCR wear may increase staining after the first night of wear, but not more than soft lenses on a longer-term basis. It is noteworthy that corneal staining was not significantly different between the two age groups. This finding suggests that the slitlamp observation of corneal response to OCR is not different in patients aged 12 years or younger compared to those older than 12 years. These data suggest that increased corneal staining compared to baseline is more related to the degree of original myopia than to age and is not predictive of adverse events. Corneal staining observed in this study was not significantly different than what has been reported with soft lenses worn on a daily-wear basis. It is noteworthy that corneal staining was recorded least often at the 1 -month visit. At 1 month in the OCR process, patients have progressed through the initial adaptation but, in general, lens deposits have not become a factor for increased corneal staining. This emphasizes that careful lens maintenance and cleaning are important factors in long-term success with OCR.

\section{Ethnicity}

Ethnicity was a significant factor in this study. Of the patients in this study, 68\% were Asian and they accounted for almost $95 \%$ of the patients aged 12 years or younger. Studies have shown that a high percentage of the Asian population become myopic at a young age. ${ }^{37}$ Similar studies in non-Asians from the United States, Scandinavia, and Australia showed myopia with less prevalence and a smaller degree. ${ }^{38}$ One recently published study ${ }^{39}$ showed the prevalence of myopia in children in rural southern China to be almost $37 \%$ by age 13 years and to increase to $54 \%$ by age 17 years. Another study of Canadian-Chinese children showed the prevalence of myopia to be $25 \%$ at age 6 years and to increase to $71 \%$ by age 12 years. ${ }^{40}$ Parents of these children are concerned about the high degree of myopia and the rate of progression in their children and look for alternatives to correction with spectacles or traditional contact lenses. The population reported in this study is similar to that in a large corneal reshaping practice in New Jersey (N. Despotidis, oral communication, 2006). The Asian population in this study showed a high degree of myopia development before the age of 12 years and interest in OCR treatment.

\section{Lens Type}

Paragon CRT lenses and custom reverse-geometry lenses were used in the study. Differences between results in using one lens versus the other are attributable to the fact that the original spherical equivalent and the original cylinder were higher for the patients who wore the custom lenses. As such, there was a greater change in spherical equivalent for the custom group versus the CRT group. Unaided acuity was insignificantly different with each lens-wearing group, but there was a significant decrease in cylinder in the custom lens group. Minor differences noted in the corneal staining between the two types of lenses also may be the result of effecting a larger change in corneal curvature and refraction with the custom-designed lenses. 


\section{Adverse Events}

Case reports of adverse events with OCR have been published ${ }^{23-30}$ in the last few years. In this study, none of the adverse events resulted in a loss of best-corrected acuity. From the current data and the case reports, a generalized statement regarding safety cannot be made. It can be said that in this setting with the given patient population, there were three adverse events during the 4.25 years observed and that these events did not cause a loss of best-corrected visual acuity. Also, the three patients involved are currently still wearing OCR lenses. After resolution of these events, modifications were made to lens design, cleaning and care regimen, and the follow-up schedule in an effort to avoid recurrence of the event. These events are serious complications and can threaten vision. They are of major concern for doctors, patients, and parents when deciding to start OCR or to continue the process once started.

Retrospectively, the treatment and care provided to these OCR patients suggests other topics of interest. First, are there axial length differences among ethnic groups in children, and secondly, does wearing OCR lenses affect changes in axial length compared to spectacle or soft lens wearers? Finally, experience from this study suggests that measurement of axial length should be standard before starting OCR. Continuing follow-up for this study population is planned to monitor longer-term corneal health, safety, and unaided visual acuity. Future study of OCR may include axial length, whether lens parameter changes are necessary over time and further evaluation of higher-order aberrations with OCR lens use.

In summary, this study showed OCR to be at least as safe and effective at temporarily reducing myopia for children aged 12 years or younger as it is for those older than 12 years. OCR is an alternative to spectacle correction or traditional contact lens correction for children and adults. OCR is of special interest to the Asian population, which develops significant myopia at a young age. It is hoped that this study, in combination with increasing experience, may help practitioners select the right patients for OCR, remain aware of potential problems and limitations, and stimulate additional research that continues to make OCR even safer and better.

\section{Acknowledgements}

The author thanks David C. Musch, Ph.D., M.S. and Leslie Niziol, M.S. of the University of Michigan Department of Ophthalmology and Visual Sciences and Department of Epidemiology for their expertise in providing the statistical data analysis.

\section{REFERENCES}

1. Walline JJ, Rah MJ, Jones LA. The Children's Overnight Orthokeratology Investigation (COOKI) pilot study. Optom Vis Sci 2004;81: 407-413.

2. Sorbara L, Fonn D, Simpson T, et al. Reduction of myopia from corneal refractive therapy. Optom Vis Sci 2005;82:512-518.

3. Paragon Vision Sciences. Premarket approval application supplement P870024/S43; June 13, 2002. Available at: http://www.fda.gov/cdrh/ pdf/P870024S0 43b.pdf. Accessed October 25, 2006.

4. Bausch \& Lomb/Euclid. Premarket approval application P010062/ S001/S002; June 7, 2004. Available at: http://www.fda.gov/cdrh/pdf/ p010062.html. Accessed October 25, 2006.
5. Alharbi A, Swarbrick HA. The effects of overnight orthokeratology lens wear on corneal thickness. Invest Ophthalmol Vis Sci 2003;44: 2518-2523.

6. Sridharan R, Swarbrick H. Corneal response to short-term orthokeratology lens wear. Optom Vis Sci 2003;80:200-206.

7. Wang J, Fonn D, Simpson TL, et al. Topographical thickness of the epithelium and total cornea after overnight wear of reverse-geometry rigid lenses for myopia reduction. Invest Ophthalmol Vis Sci 2003;44: $4742-4746$.

8. Choo J, Caroline P, Harlin D. How does the cornea change under corneal reshaping lenses? Eye Contact Lens 2004;30:211-213.

9. Hiraoka T, Okamoto F, Kaji Y, et al. Optical quality of the cornea after overnight orthokeratology. Cornea 2006;25 (suppl 1):S59-S63.

10. Joslin C, Wu S, McMahon T, et al. Higher-order wavefront aberrations in corneal refractive therapy. Optom Vis Sci 2003;80:805-811.

11. Berntsen D, Barr JT, Mitchell GL. The effect of overnight contact lens corneal reshaping on higher-order aberrations and best-corrected visual acuity. Optom Vis Sci 2005;82:490-497.

12. Johnson KL, Carney LG, Mountford JA, et al. Visual performance after overnight orthokeratology. Contact Lens Anterior Eye 2007;30: 29-36.

13. Barr JT, Rah MJ, Jackson JM, et al. Orthokeratology and corneal refractive therapy: A review and recent findings. Eye Contact Lens 2003;29 (suppl 1):S49-S53.

14. Soni S, Nguyen T. Overnight orthokeratology experience with XO material. Eye Contact Lens 2006;32:39-45.

15. Lipson M, Sugar A, Musch D. Overnight corneal reshaping versus soft disposable contact lenses: Vision-related quality of life differences from a randomized clinical trial. Optom Vis Sci 2005;82:886-891.

16. Berntsen DA, Mitchell GL, Barr JT. The effect of overnight contact lens corneal reshaping on refractive error-specific quality of life. Optom Vis Sci 2006;83:354-359.

17. Soni S, Nguyen T, Bonanno J. Overnight orthokeratology: Refractive and corneal recovery after discontinuation of reverse-geometry lenses. Eye Contact Lens 2004;30:254-262.

18. Barr J, Rah M, Meyers W, et al. Recovery of refractive error after corneal refractive therapy. Eye Contact Lens 2004;30:247-251.

19. Cho P, Cheung SW, Edwards M. The Longitudinal Orthokeratology Research in Children (LORIC) in Hong Kong: A pilot study on refractive changes and myopia control. Curr Eye Res 2005;30:71-80.

20. Fan L, Jun J, Jia Q, et al. Clinical study of orthokeratology in young myopic adolescents. Int Contact Lens Clin 1999;26:113-116.

21. Rah M, Bailey M, Hayes J, et al. Comparison of NEI RQL-42 Scores in LASIK vs. CRT patients [abstract]. Invest Ophthalmol Vis Sci 2004;45:E-Abstract 1578.

22. Schein OD, Buehler PO, Stamler JF, et al. The impact of overnight wear on the risk of contact lens-associated ulcerative keratitis. Arch Ophthalmol 1994;112:186-190.

23. Nichols J, Marsich M, Nguyen M, et al. Overnight orthokeratology. Optom Vis Sci 2000;77:252-259.

24. Walline JJ, Holden BA, Bullimore MA, et al. The current state of corneal reshaping. Eye Contact Lens 2005;31:209-214.

25. Watt K, Swarbrick HA. Microbial keratitis in overnight orthokeratology: Review of the first 50 cases. Eye Contact Lens 2005;31: 201-208.

26. Tseng C-H, Fong C-F, Chen W-L, et al. Overnight orthokeratology associated microbial keratitis. Cornea 2005;24:778-782.

27. Hsiao C-H, Lin H-C, Chen Y-F, et al. Infectious keratitis related to overnight orthokeratology. Cornea 2005;24:783-788.

28. Schein OD. Microbial keratitis associated with overnight orthokeratology: What we need to know. Cornea 2005;24:767-769.

29. Macsai MS. Corneal ulcers in two children wearing Paragon Corneal Refractive Therapy lenses. Eye Contact Lens 2005;31:9-11.

30. Lang J, Rah M. Adverse corneal events associated with corneal reshaping: A case series. Eye Contact Lens 2004;30:231-233.

31. Saviola JF. The current FDA view on overnight orthokeratology: How we got here and where we are going. Cornea 2005;24:770-771.

32. Norman C. Current research in corneal reshaping. Contact Lens Spectrum 2005. http://www.clspectrum.com/article.aspx?article $=12890$.

33. Schwallie JD, McKenney CD, Long WD, et al. Corneal staining patterns in normal non-contact lens wearers. Optom Vis Sci 1997;74: 92-98. 
34. Dundas M, Walker A, Woods R. Clinical grading of corneal staining of non-contact lens wearers. Ophthalmic Physiol Opt 2001;21:30-35.

35. Begley CG, Barr JT, Edrington TB, et al. Characteristics of corneal staining in hydrogel contact lens wearers. Optom Vis Sci 1996;73:193-200.

36. Nichols K, Mitchell GL, Stonebraker K, et al. Corneal staining in hydrogel lens wearers. Optom Vis Sci 2002;79:20-30.

37. Seet B, Wong TY, Tan DTH, et al. Myopia in Singapore: Taking a public health approach. Br J Ophthalmol 2001;78:234-239.
38. Kempen J, Mitchell P, Lee KE, et al. The prevalence of refractive errors among adults in the United States, Western Europe, and Australia. Arch Ophthalmol 2004;122:495-505.

39. He M, Huang W, Zheng Y, et al. Refractive error and visual impairment in school children in rural southern China. Ophthalmology 2007;114:374-382.

40. Cheng D, Schmid K, Woo G. Myopia prevalence in ChineseCanadian children in an optometric practice. Optom Vis Sci 2007; 84:21-32. 